\title{
EXTENDED-STORAGE IRREVERSIBLE HYDROCOLLOID IMPRESSION MATERIALS
}

\author{
Muhammed B. El-Danasory $B D S^{1}$, Muhammed A. Gad $P h D^{2}$, Seham A. Hanafy $P H D^{3}$
}

\begin{abstract}
INTRODUCTION: There are many contingencies, so many dentists do not pour their own impressions immediately which clears the need for extended-storage alginate impression materials.

OBJECTIVES: Evaluating and comparing three extended-storage alginate impression materials concerning their dimensional stability.

MATERIALS AND METHODS: One hundred and eighty (180) specimens were prepared in accordance with ADA specification No. 18 using three extended-storage alginates; Hydrogum5 (Zhermack), Cavex ColorChange (Cavex) and Blueprint X Crème (Dentsply). Following to ADA specification No. 19 dimensional stability was evaluated directly at six intervals; 0, 24, 48, 72,96 and 120 hours using 10 specimens/interval /material. Specimens for delayed evaluation, were stored moistened within sealed plastic bags in a dark room at $23 \pm 1^{\circ} \mathrm{c}$. RESULTS: All impression materials showed initial expansion and ended with a net shrinkage. Cavex ColorChange showed the least dimensional changes. Dimensional changes of Cavex ColorChange, Hydrogum5 and Blueprint Xcrème ranged (0.14 - 0.59, 0.37 - 2.07 and $0.35-1.40 \%$ respectively).

CONCLUSION: While Cavex ColorChange can be stored up to 120 hours without clinically significant dimensional changes, Hydrogum5 and Blueprint Xcrème should be poured immediately for the best results.

KEYWORDS: Alginate; Extended storage; Dimensional stability; Cavex ColorChange; Hydrogum5; Blueprint Xcrème.
\end{abstract}

1- Instructor at the removable prosthodontics department, faculty of Dentistry, Alexandria University, Alexandria, Egypt.

2- Professor of Prosthodontics, faculty of Dentistry, Alexandria University, Alexandria, Egypt.

3- Professor of Dental Material, faculty of Dentistry, Alexandria University, Alexandria, Egypt.

\section{INTRODUCTION}

Alginate has been the staple of most dental practices for many years. The general use of irreversible hydrocolloid impression materials far exceeds that of any impression material, because of their various advantages such as hydrophilicity, pleasant taste and odor, non-staining, inexpensive and ease of mixing. However they have low tear strength and dimensional stability (1).

As with any hydrocolloid, alginates are prone to distortion caused by expansion associated with imbibition (absorption of moisture) or shrinkage due to moisture loss by evaporation, or continued reaction (syneresis) (2).

Casts produced from alginate impressions must be generated immediately or within

3 hours after the impressions are removed from the patient's mouth (3). There are many contingencies so, many dentists do not pour their impressions immediately and send them to the commercial laboratories. Under these circumstances, a considerable delay occurs between the removal of the impression from the mouth, and the eventual pouring of the cast which increases the incidence of dimensional changes (4).

The problem which clears the need for the extendedstorage alginate impression material. They have been marketed with claims that they have good dimensional stability and can be poured even after 100 hours without significant changes (5).

In the available literature, many studies have been made to evaluate the dimension stability of the extended-storage alginate impression materials. The results have led to conflicting conclusions where some of them reinforced the claims of 5-day dimension stability while most of them recommended pouring the alginate impressions within 5 days or even immediately (4-9).

In addition, most studies have evaluated the dimensional stability indirectly via measurements on gypsum casts, which incorporate the confounding variable of gypsum expansion $(4,7,9)$.
Therefore, the purpose of this study is to directly evaluate and compare three extended-storage alginate impression materials concerning their dimensional stability over 120 hours.

\section{MATERIAL AND METHOD}

Three extended-storage alginate impression materials; Hydrogum5 (Zhermack, Badia, Polesine, Italy), Cavex ColorChange (Cavex Holland BV, Haarlem, The Netherlands) and Blueprint X Crème (Dentsply Caulk, Milford, DE, USA).

\section{Specimen preparation}

Specimen preparation followed the protocol of ADA Specification No. 18 for alginate impression materials (10).

Each alginate was evacuated from its package into a sealed container to allow through fluffing of the alginate to loosen the condensed powder and to ensure even distribution of its components. The container was being fluffed 4 times before each scooping. Using the manufacturer's recommended W/P ratio (Table1) the alginate powder was mixed with distilled water by hand mixing using a flexible rubber bowel and straight plastic spatula.

Table (1): Shows the $\mathrm{W} / \mathrm{P}$ ratio $(\mathrm{ml} / \mathrm{g})$ and setting time of different types of alginates as the manufacture recommends.

\begin{tabular}{|l|l|l|l|}
\hline Item & $\begin{array}{l}\text { Hydrogu } \\
\text { m5 }\end{array}$ & $\begin{array}{l}\text { Cavex } \\
\text { Color } \\
\text { Change }\end{array}$ & $\begin{array}{l}\text { Blueprint } \\
\text { X-Crème }\end{array}$ \\
\hline W/P Ratio (ml/g) & 2.14 & 2.17 & 2.14 \\
\hline $\begin{array}{l}\text { Setting Time } \\
\text { (min) }\end{array}$ & $2 ' 20 "$ & $2^{\prime} 30 "$ & $2^{\prime \prime} 30^{\prime \prime}$ \\
\hline
\end{tabular}


The mixed alginate was placed into the mold specified for the test and the excess was wiped using a glass slab. Then, the mold was placed into a bath of distilled water maintained at $35 \pm 1^{\circ} \mathrm{C}$ and loaded with a $1 \mathrm{~kg}$ mass conditioned at the same temperature.

Three minutes after the manufacturer's stated setting time (Table1) the assembly was removed from the water bath and the set alginate specimen was separated from the metal die. After specimen removal, it was rinsed with distilled water to simulate rinsing following impression removal from the mouth and the excess fluid was shaken off. The specimens for delayed evaluation were stored at $23 \pm 1{ }^{\circ} \mathrm{C}$ in sealed plastic bag in a dark room.

\section{Dimensional stability}

The specification for irreversible hydrocolloid impression materials (ADA NO. 18) does not include an evaluation protocol for dimensional change (10). Therefore the dimensional stability was evaluated according to ADA Specification No. 19 for dental elastomeric impression materials (11).

A copper die was used for making the specimens. The die (Fig.1) consists of 2 parts: a test block and a mold. The test block has 3 lines that are crossed with 2 perpendicular lines.

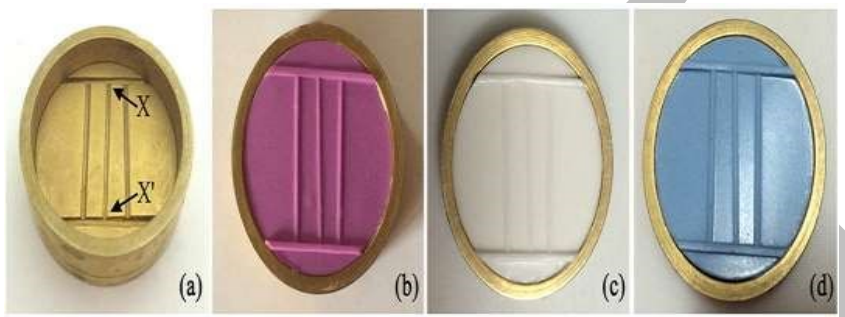

Figure (1): The test block \& mold (a), hydrogum 5 (b), caver color change, (c) blueprint xcreme (d).

The dimension stability was evaluated by comparing the length of the middle line of the copper die $\left(\mathrm{X}^{\prime} \mathrm{X}^{\prime}\right)$ with the same line in the specimen. The measurements were directly made via toolmaker's microscope (Carl Zeiss, Jena, Germany, Fig. 2).

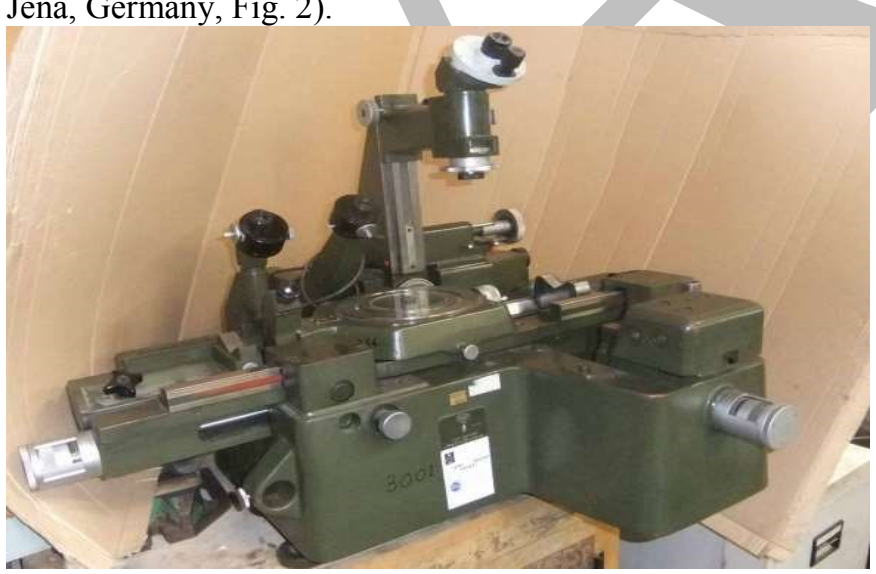

Figure (2): Toolmaker's microscope.

The percentage of the dimensional change of the specimen from the copper die was calculated using the following equation:

The dimensional change $=[(\mathrm{A}-\mathrm{D}) / \mathrm{D}] \mathrm{X} 100$

A: The specimen measurement.
D: The die measurement $(23.51 \mathrm{~mm})$. specimens were made for each of the following intervals $0,24,48,72,96$ and 120 hours after the setting of the impression.

\section{Statistical analyses}

Data were fed to a computer and analyzed using IBM SPSS software package version 20.0. Significance (P) of the obtained results was judged at the $5 \%$ level. Stars $\left(^{*}\right)$ were used to highlight significant values within the table. $\mathrm{P}_{0}$ is the significance value of One-Sample- $\mathrm{T}$ test to compare the intervals within a material with $0 \%$ dimensional change while $\mathrm{P}_{1}, \mathrm{P}_{2}$ and $\mathrm{P}_{3}$ are the significance value of Mann Whitney test to compare the materials with each other where:

- $\mathrm{P}_{1}$ is $\mathrm{p}$ value for comparing between Hydrogum5 and Cavex ColorChange.

- $\mathrm{P}_{2}$ is $\mathrm{p}$ value for comparing between Hydrogum5 and Blueprint X-Crème.

- $\mathrm{P}_{3}$ is $\mathrm{p}$ value for comparing between Cavex ColorChange and Blueprint X-Crème.

\section{RESULTS}

(Table2) demonstrates the values of the mean (M) and standard of deviation (SD.) of the calculated dimensional changes of the three alginate impression materials across the period $0-120$ hours at six intervals.

All the intervals within a material showed a statistically significant changes from the master model except the second interval (24 hours) for Cavex ColorChange. Moreover, all the materials presented an initial expansion at the first interval ( 0 hours) and a net contraction at the sixth interval (120-hours). Cavex ColorChange is the only one that kept the expansion till the second interval (24-hours).

Also it is worth to be mentioned that on comparing the materials with each other, the dimensional changes at the first interval ( 0 hours) are statistically insignificant while they are significant for the other intervals.

Hydrogum5 and Blueprint X-Crème presented the minimal amount of dimensional change at the first interval (0 hours) while Cavex ColorChange showed the minimal change at the second interval ( 24 hours). For Hydrogum5, Cavex ColorChange and Blueprint X-Crème, the maximum changes were observed at 72 hours, 0 hours and 120 hours respectively. (Fig.3)

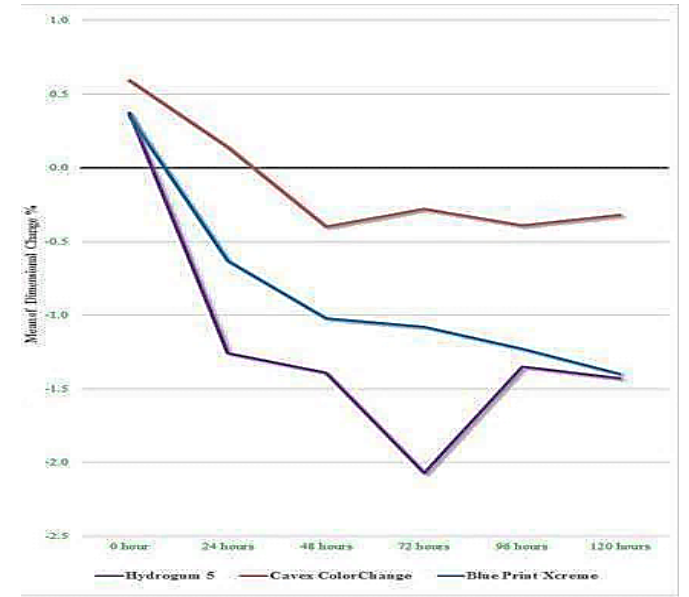

Figure (3): Shows the changes of the dimensional changes $\%$ of the three alginate impression materials across the intervals. 
Table (2): Shows the values of M (SD.) of the dimensional changes $\%$ of the three alginate impression materials across the intervals, $\mathrm{n}=10$ for each interval.

\begin{tabular}{|c|c|c|c|c|c|c|c|}
\hline \multicolumn{2}{|c|}{ Material } & $\mathbf{0 h}$ & $24 \mathrm{~h}$ & $48 \mathrm{~h}$ & $72 \mathrm{~h}$ & $96 h$ & $120 \mathrm{~h}$ \\
\hline \multirow{2}{*}{ Hydrogum5 } & M (SD.) & $0.37(0.23)$ & $-1.26(0.36)$ & $-1.39(0.34)$ & $-2.07(0.39)$ & $-1.35(0.37)$ & $-1.43(0.32)$ \\
\hline & po & $0.001^{*}$ & $<0.001^{*}$ & $<0.001^{*}$ & $<0.001^{*}$ & $<0.001^{*}$ & $<0.001^{*}$ \\
\hline \multirow{2}{*}{ Cavex ColorChange } & M (SD.) & $0.59(0.27)$ & $0.14(0.23)$ & $-0.40(0.46)$ & $-0.28(0.25)$ & $-0.39(0.36)$ & $-0.32(0.20)$ \\
\hline & po & $<0.001^{*}$ & 0.082 & $0.021^{*}$ & $0.007^{*}$ & $0.007^{*}$ & $0.001^{*}$ \\
\hline \multirow{2}{*}{$\begin{array}{l}\text { Blueprint } \\
\text { X-Crème }\end{array}$} & M (SD.) & $0.35(0.29)$ & $-0.63(0.19)$ & $-1.02(0.28)$ & $-1.08(0.34)$ & $-1.23(0.41)$ & $-1.40(0.36)$ \\
\hline & po & $0.004^{*}$ & $<0.001^{*}$ & $<0.001^{*}$ & $<0.001^{*}$ & $<0.001^{*}$ & $<0.001^{*}$ \\
\hline \multicolumn{2}{|l|}{$\mathbf{P}_{1}$} & 0.096 & $<0.001^{*}$ & $0.001^{*}$ & $<0.001^{*}$ & $<0.001^{*}$ & $<0.001^{*}$ \\
\hline \multicolumn{2}{|l|}{$\mathbf{P}_{2}$} & 0.762 & $0.001^{*}$ & $0.025^{*}$ & $<0.001^{*}$ & 0.404 & 1.000 \\
\hline \multicolumn{2}{|l|}{$\mathbf{P}_{3}$} & 0.064 & $<0.001^{*}$ & $0.006^{*}$ & $<0.001^{*}$ & $0.001^{*}$ & $<0.001^{*}$ \\
\hline
\end{tabular}

\section{DISCUSSION}

Almost all fields of dental practice use the alginate impression materials for either diagnosis and treatment planning or fabrication of restorations $(7,12-14)$ as they have many advantages (1). The motive that has encouraged the companies for dental materials all over the world to enhance the properties of the alginates and treat their deficiencies.

These efforts have resulted in new products of the alginate impression materials with claims of being dimensionally stable up to 5 or 9 days and were named extendedstorage alginate impression materials which evoked much controversies (4-9).

Sixty specimens per material were prepared where ten specimens were evaluated for each interval and discarded as it was important to make the results as clinically applicable as possible, and exposing the impression to air multiple times doesn't occur in dental practices.

Toolmaker's microscope provides accuracy up to $10 \mu \mathrm{m}$ which is clinically acceptable especially when the mean value of the marginal gap between the fixed restorations and their abutments is $81-120 \mu \mathrm{m}(15)$.

All the studied impression materials showed initial expansion which may be explained as following: an osmotic potential was created due to the gradient concentration of the ions between the alginate specimens and the water that remained on them on storing. This osmotic potential resulted in water absorption and so the expansion was continuing till equalization of the ions concentration (2).

Cavex ColorChange is the only one that kept the expansion at the 24 hours, the result which can be owed to its much higher initial expansion in comparison with Hydrogum 5 and Blueprint Xcrème at 0 hours.

For all studied materials, the expansion was followed by shrinkage which can be, mainly, owed to syneresis that was recognized with a greasy exudate covering the specimens on getting them out of the plastic bags. Also shrinkage may be increased by the reversed osmotic potential resulted from diffusion of the ions outside the specimens (2). Evaporation is another factor but it was minimized through the storing conditions.

The shrinkage of the specimens occurred toward its center so, the linear measurements was reduced. In a clinical situation during full-arch impressions, when the impression is firmly bonded to the tray, shrinkage will result in the impression material being pulled toward the tray, causing an increase of the dimensions of the teeth due to the centrifugal tensile forces and decreased cross arch palatal dimensions (7).

The obtained results of Hydrogum5 and Blueprint Xcrème at the first interval ( 0 hours) and these of Cavex ColorChange at all intervals ( 0 - 120 hours $)$ are coincident with ADA No. 19 (11).

Moreover, when Hydrogum5 and Blueprint Xcrème are stored $24-120$ hours, they showed shrinkage ranging ( 1.26 to $2.07,0.63$ to $1.40 \%$ ) respectively, however this shrinkage is not clinically significant for diagnostic casts and for fabrication of acrylic appliances.

The differences between the studied materials may be explained by the different composition where it was found that alginates with a higher ratio of calcium to sodium exhibit greater dimensional stability (16). In addition, the molecular weight of the alginate chains, different W/P ratios and additives like the color indicator that controls the $\mathrm{pH}$ may provide an explanation.

Imbery TA (7) obtained initial shrinkage of Cavex ColorChange followed with expansion which decreased with the storage period and while Sedda (2008) (4) and Rohanian (2014) (9) recorded shrinkage of Hydrogum5 at the different intervals, Mosharraf (8) found expansion. Although they reported insignificant dimensional changes of the studied impression materials, they recommended immediate pouring for accurate casts.

These contrasting results may be occurred due to the different mixing technique, the storing conditions and number of specimens or because they used modified models which introduce the elastic recovery as a factor. Also they evaluated the dimensional stability indirectly on poured casts which introduces the factor of the dimensional stability of the dental stone.

\section{CONCLUSIONS}

- Over 120 hours, Cavex ColorChange has much better dimensional stability than Hydrogum5 and Blueprint $\mathrm{X}$ crème which showed close results.

- For producing casts coincident with ADA specifications No. 19 regarding the dimensional stability, Cavex ColorChange can be poured any time 
up to 120 hours while Hydrogum5 and Blueprint Xcrème should be poured as soon as possible.

- When Hydrogum5 and Blueprint Xcrème are stored 24 - 120 hours, they can be used for fabrication of diagnostic casts and acrylic appliances.

\section{CONFLICT OF INTEREST}

The authors declare that they have no conflicts of interest

\section{REFERENCES}

1. Carr AB, McGivney GP, Brown DT. McCracken's Removable partial prosthodontics, 12th ed, St. Louis: Mosby; 2010.

2. Nallamuthu NA, Braden M, Patel MP. Some aspects of the formulation of alginate dental impression materials-Setting characteristics and mechanical properties. J Dental Materials. 2012;28:756-62.

3. Dahl LB, Dymbe B, Valderhaug J. Bonding properties and dimensional stability of hydrocolloid impression systems in fixed prosthodontics. J Prosthet Dent. 1985;53:796-800.

4. Sedda M, Casarotto A, Rausita A, Borracchini A. Effect of storage time on the accuracy of casts made from different irreversible hydrocolloids. J Contemporary Dental Practice. 2008;9:59-66.

5. Walker MP, Burckhard J, Mitts DA, Williams KB. Dimensional change over time of extended-storage alginate impression materials. Angle Orthod. 2010;80:1110-15.

6. Rodrigues SB, Augusto CR, Leitune VCB, Samuel SMW, Collares FM. Influence of delayed pouring on irreversible hydrocolloid properties. J Brazilian Oral Research (São Paulo). 2012;26:404-9.

7. Imbery TA, Nehring J, Janus C, Moon PC. Accuracy and dimensional stability of extended-pour and conventional alginate impression materials. J Am Dent Assoc. 2010;141:32-9.

8. Mosharraf R, Nosouhian S, Salehi M. Effect of storage time on dimensional stability of Extended-pour irreversible hydrocolloid impression material. J Isfahan Dental School. 2011;7:246-55.

9. Rohanian A, Shabestari GO, Zeighami S, Samadi MJ, Shamshiri AR. Effect of Storage Time of ExtendedPour and Conventional Alginate Impressions on Dimensional Accuracy of Casts. J Dentistry (Tehran). 2014;11:655-64.

10. Revised American National Standards Institute/American Dental Association, ANSI/ADA, Specification No. 18 for dental alginate impression materials, Chicago Ill: ADA; 1992.

11. American National Standard Institute/American Dental Association, ANSI/ADA, Specification No. 19 for Dental Elastomeric Impression Materials, Chicago Ill: ADA; 2004.

12. Fernandes A, Dua N, Herekar M. Corrective Primary Impression Technique. Open Dental Journal. 2010;4:27-8.

13. Kitamura A, Kawai Y. Basic investigation of the laminated alginate impression technique: Setting time, permanent deformation, elastic deformation, consistency, and tensile bond strength tests. J Prosthodontic Research. 2015;59:49-54.
14. Eriksson A, Ockert-Eriksson G, Lockowandt P. Accuracy of irreversible hydrocolloids (alginates) for fixed prosthodontics. A comparison between irreversible hydrocolloid, reversible hydrocolloid, and addition silicone for use in the syringe-tray technique. Eur J Oral Sci. 1998;106:651-60.

15. Bayramoğlu E, Özkan YK, Yildiz C. Comparison of marginal and internal fit of presson-metal and conventional ceramic systems for three- and four-unit implant-supported partial fixed dental prostheses: An in vitro study. J Prosthet Dent. 2015;114:52-8.

16. Fellows CM, Thomas GA. Determination of bound and unbound water in dental alginate irreversible hydrocolloid by nuclear magnetic resonance spectroscopy. J Dental Materials. 2009;25:486-93. 